\title{
Diagnosing ions and neutrals via $n=2$ excited hydrogen atoms in plasmas with high electron density and low electron temperature
}

Citation for published version (APA):

Shumack, A. E., Schram, D. C., Biesheuvel, J., Goedheer, W. J., \& Rooij, van, G. J. (2011). Diagnosing ions and neutrals via $\mathrm{n}=2$ excited hydrogen atoms in plasmas with high electron density and low electron temperature. Physical Review E - Statistical, Nonlinear, and Soft Matter Physics, 83(3), 036402-12. [036402]. https://doi.org/10.1103/PhysRevE.83.036402

DOI:

10.1103/PhysRevE.83.036402

Document status and date:

Published: 01/01/2011

Document Version:

Publisher's PDF, also known as Version of Record (includes final page, issue and volume numbers)

\section{Please check the document version of this publication:}

- A submitted manuscript is the version of the article upon submission and before peer-review. There can be important differences between the submitted version and the official published version of record. People interested in the research are advised to contact the author for the final version of the publication, or visit the $\mathrm{DOI}$ to the publisher's website.

- The final author version and the galley proof are versions of the publication after peer review.

- The final published version features the final layout of the paper including the volume, issue and page numbers.

Link to publication

\footnotetext{
General rights

- You may freely distribute the URL identifying the publication in the public portal. follow below link for the End User Agreement:

www.tue.nl/taverne

Take down policy

If you believe that this document breaches copyright please contact us at:

openaccess@tue.nl

providing details and we will investigate your claim.
}

Copyright and moral rights for the publications made accessible in the public portal are retained by the authors and/or other copyright owners and it is a condition of accessing publications that users recognise and abide by the legal requirements associated with these rights.

- Users may download and print one copy of any publication from the public portal for the purpose of private study or research.

- You may not further distribute the material or use it for any profit-making activity or commercial gain

If the publication is distributed under the terms of Article 25fa of the Dutch Copyright Act, indicated by the "Taverne" license above, please 


\title{
Diagnosing ions and neutrals via $n=2$ excited hydrogen atoms in plasmas with high electron density and low electron temperature
}

\author{
A. E. Shumack, D. C. Schram, J. Biesheuvel, W. J. Goedheer, and G. J. van Rooij \\ FOM-Institute for Plasma Physics Rijnhuizen, Association EURATOM-FOM, Trilateral Euregio Cluster, Nieuwegein, The Netherlands
}

(Received 31 October 2010; revised manuscript received 8 December 2010; published 2 March 2011)

\begin{abstract}
Ion and neutral parameters are determined in the high electron density, magnetized, hydrogen plasma beam of an ITER divertor relevant plasma via measurements of the $n=2$ excited neutrals. Ion rotation velocity (up to $7 \mathrm{~km} / \mathrm{s}$ ) and temperature $\left(2-3 \mathrm{eV} \sim T_{e}\right.$ ) are obtained from analysis of $\mathrm{H} \alpha$ spectra measured close to the plasma source. The methodology for neutral density determination is explained whereby measurements in the linear plasma beam of Pilot-PSI are compared to modeling. Ground-state atomic densities are obtained via the production rate of $n=2$ and the optical thickness of the Lyman- $\alpha$ transition (escape factor $\sim 0.6$ ) and yield an ionization degree $>85 \%$ and dissociation degree in the residual gas of $\sim 4 \%$. A $30 \%$ proportion of molecules with a rovibrational excitation of more than $2 \mathrm{eV}$ is deduced from the production rate of $n=2$ atoms. This proportion increases by more than a factor of 4 for a doubling of the electron density in the transition to ITER divertor relevant electron densities, probably because of a large increase in the production and confinement of ground-state neutrals. Measurements are made using laser-induced fluorescence (LIF) and absorption, the suitability of which are evaluated as diagnostics for this plasma regime. Absorption is found to have a much better sensitivity than LIF, mainly owing to competition with background emission.
\end{abstract}

DOI: 10.1103/PhysRevE.83.036402

PACS number(s): $52.70 . \mathrm{Kz}, 52.25 . \mathrm{Os}, 52.65 . \mathrm{Pp}, 52.25$.Ya

\section{INTRODUCTION}

The plasma conditions expected in the divertor of the experimental fusion reactor ITER constitute a new regime in plasma physics. The high electron density $\left(\sim 10^{20} \mathrm{~m}^{-3}\right)$ ensures that the (hydrogen) plasma is not transparent for neutrals (produced by recombination at the divertor plates) and the low electron temperature $(\sim 1-5 \mathrm{eV})$ is such that the plasma balances on the boundary between an ionizing and recombining plasma. Especially, the interaction between charged and neutral species in this plasma regime is not trivial. For the progression of plasmawall interaction studies in fusion research [1], a good understanding of this plasma regime is imperative. Quantities that need to be measured to achieve this are density, temperature, and velocity of electrons, protons, atoms, and (rovibrationally excited) molecules. Electron temperature and density can be easily measured with excellent statistics in a high electrondensity plasma using Thomson scattering [2,3]. Protons and ground-state atoms are more difficult to access nonintrusively.

More readily accessible via active spectroscopy are $n=2$ excited atoms. In this paper we analyze the production and destruction processes of the $n=2$ excited-atom population (Sec. II) and show how we can use this species in an ITER divertor relevant regime to access density information on ground-state atoms and rovibrationally excited molecules and temperature and velocity information on protons.

Experiments are performed in the magnetized plasma beam of Pilot-PSI (described in Sec. III), a linear, magnetized hydrogen plasma generator. This machine simulates the energy flux to the divertor plates expected in ITER. It also uniquely produces plasmas of such high electron density (at temperatures of a few eV) that-as expected in the divertor of ITER-interaction between ground-state atoms and ions is important and rovibrationally excited molecules have very short mean free paths (of only a few millimeters).

One major difference between the plasma of Pilot-PSI and the ITER divertor is the fact that the beam of Pilot-PSI is surrounded by gas. Rovibrationally excited molecules will penetrate the edge of the beam and play a significant role in the plasma dynamics. For high electron-density conditions (ITER divertor relevant electron densities), these molecules will not reach the center of the beam. The dynamics at a distance from the divertor plates in ITER are therefore well simulated in the center of the beam of Pilot-PSI. Another difference between Pilot-PSI and the ITER divertor concerns the surface area of the impinging beam. To properly study the plasma at the ITER divertor plates, the beam should be wider than the mean free paths of the most important processes. This is not necessarily the case for the $\sim 1$-cm-diam beam of Pilot-PSI.

The results presented in this paper are measured in both the central and edge regions of the plasma beam. They are relevant for the understanding of linear divertor simulators in general, as well as (especially in the case of information about the center of the beam) for understanding the ITER divertor itself. This paper also forms a framework for future measurements in the upgrade of Pilot-PSI-Magnum-PSI [4], in which the plasma beam diameter will be larger and the effect of background molecules therefore will be less important.

The parameters that are varied in this paper are electron density, background pressure, and lateral position. We study the density behavior of various neutral populations in the transition from the weakly magnetized, low electron density, hydrogen plasma regime [5-8] up to ITER divertor relevant electron densities. The effect of background pressure is explored by varying the background pressure from low $(\sim 6.5 \mathrm{~Pa}$, i.e., close to the $1 \mathrm{~Pa}$ neutral pressure expected in the ITER divertor) to high $(\sim 50 \mathrm{~Pa})$. Lateral scans across the diameter of the beam allow study of the penetration of molecules into the beam. Measurements are made at a large distance from the target, so that in the interpretation recycling effects at the target need not be considered. An overview of the plasma conditions studied in this paper is given in Sec. III B.

We measure the $n=2$ atoms using both laser-induced fluorescence (LIF) and absorption spectroscopy. The data 
analysis is outlined in Sec. III C and results are presented in Sec. IV. Ground-state atom densities are estimated from these measurements via a Monte Carlo simulation of the reabsorption of Lyman- $\alpha$ photons (Sec. V). Other neutral densities are determined in Sec. VI by comparison of measurements with the population balance. The suitabilities of the two diagnostic techniques in the given plasma regime are compared in the discussion (Sec. VII), together with a discussion of the results on ion parameters. Conclusions are given in Sec. VIII.

\section{ANALYSIS OF THE POPULATION BALANCE OF $n=2$ EXCITED ATOMS}

In this section, we identify the most dominant production and destruction mechanisms for $n=2$ excited neutrals. This will serve as a framework for understanding the measurement data. We set up a local model in order to predict the $n=2$ density that can be expected at a single position depending on densities of other species at that same position.

\section{A. Production mechanisms of $\boldsymbol{n}=\mathbf{2}$ excited neutrals}

The dominant population processes for $n=2$ in our plasma are molecular activated recombination (MAR) and relaxation (via electron deexcitation and radiation) from the $n=3$ level, which is itself dominantly populated by MAR. MAR is charge exchange (c.x.),

$$
\mathrm{H}_{2}(v \geqslant 4)+\mathrm{H}^{+} \rightarrow \mathrm{H}_{2}^{+}\left(v^{\prime}\right)+\mathrm{H},
$$

followed by dissociative recombination (d.r.),

$$
\mathrm{H}_{2}{ }^{+}\left(v^{\prime}\right)+e^{-} \rightarrow \mathrm{H}^{*}+\mathrm{H} .
$$

(See Refs. [6], [9], and [10].) $\mathrm{H}_{2}(v \geqslant 4)$ is a rovibrationally excited hydrogen molecule, with an excitation energy larger than $2 \mathrm{eV} . \mathrm{H}^{*}$ indicates an excited hydrogen atom. The distribution of excited states from reaction (2) is assumed to be the same as for d.r. with $v=0-9(10 \% n=2,45 \%$ $n=3,22 \% n=4,23 \% n>4)[11])$. Above $n_{e}=10^{19} \mathrm{~m}^{-3}$, charge exchange is the slowest of the two reactions and thus rate determining. Above $1 \mathrm{eV}$, the rate coefficient $\langle\sigma v\rangle$ of charge exchange is $3 \times 10^{-15} \mathrm{~m}^{3} \mathrm{~s}^{-1}$ [12] for molecules with a vibrational excitation $v \geqslant 4$. For molecules with a lower vibrational energy, the rate coefficients are $\sim 100$ times lower.

Other direct $n=2$ and $n=3$ production mechanisms are less important in our plasma. Direct electron excitation to $n=$ 2 has a total rate coefficient of $8 \times 10^{-16} \mathrm{~m}^{3} \mathrm{~s}^{-1}$ at $3 \mathrm{eV}$ [11] (decreasing fast at lower electron temperatures) and is slower for $n=3$. It may give a minor contribution to the population of the $n=2$ level, however, we will show experimentally that it can be ignored. The net effect of three-particle recombination $\left(\langle\sigma v\rangle<2.9 \times 10^{-40} \mathrm{~m}^{6} \mathrm{~s}^{-1}[13]\right.$ for $\left.T_{e}>1 \mathrm{eV}\right)$, corrected for by loss via direct ionization, only gives a significant contribution to $n=2$ and $n=3$ levels for electron densities higher than $7 \times 10^{21} \mathrm{~m}^{-3}$. Mutual neutralization of $\mathrm{H}^{-}$and $\mathrm{H}^{+}$has a high rate coefficient $\left(\sim 3 \times 10^{-15} \mathrm{~m}^{3} \mathrm{~s}^{-1}\right.$ for $n=2$ and $5 \times 10^{-14} \mathrm{~m}^{3} \mathrm{~s}^{-1}$ for $n=3$ ), but at high electron density, there are a multitude of competing processes involving $\mathrm{H}^{-}$. Dissociative recombination of $\mathrm{H}_{3}^{+}$to $n=2$ also has a high rate coefficient $\left(\sim 3 \times 10^{-14} \mathrm{~m}^{3} \mathrm{~s}^{-1}\right)$, but requires $\mathrm{H}_{2}+$ ions for production, the density of which is kept low by dissociative recombination and mutual neutralization. We thus conclude that MAR is the dominant direct excitation mechanism for both $n=2$ and $n=3$. We also include relaxation to $n=2$ from the level closest by $n=3$. Estimations have shown that relaxation from other levels to $n=2$ and $n=3$ levels can be ignored.

The rate of MAR is $\alpha n_{e} n_{\mathrm{H}_{2}}+k_{\mathrm{dr}}$, where $n_{e}$ is the electron density $\left(\mathrm{m}^{-3}\right), k_{\mathrm{dr}}$ is the rate of dissociative recombination $\left(\mathrm{m}^{3} \mathrm{~s}^{-1}\right)$ (all rate coefficients used in this paper are taken from Ref. [11] unless stated otherwise), $\alpha$ is the branching ratio for the relevant excited state $(0.1$ for $n=2$ and 0.45 for $n=3[11])$, and $n_{\mathrm{H}_{2}}\left(\mathrm{~m}^{-3}\right)$ is the density of $\mathrm{H}_{2}{ }^{+}$calculated from the balance

$$
\begin{aligned}
\frac{d n_{\mathrm{H}_{2}^{+}}}{d t}= & 0=n_{\mathrm{H}^{+}} n_{\mathrm{H}_{2}(v \geqslant 4)} k_{\mathrm{cx}} \\
& -n_{\mathrm{H}_{2}}\left[n_{e} k_{\mathrm{dr}}+n_{\mathrm{H}_{2}}\left(k_{\mathrm{H}_{3}+}+k_{\mathrm{cx}_{2}}\right)\right],
\end{aligned}
$$

where $k_{\mathrm{cx}}$ is the rate of charge exchange (reaction (1)), $k_{\mathrm{cx}_{2}}$ is the rate of the charge exchange reaction between $\mathrm{H}_{2}^{+}$and $\mathrm{H}_{2}$, and $k_{\mathrm{H}_{3}}$ is the rate of the $\mathrm{H}_{3}{ }^{+}$producing reaction [13]

$$
\mathrm{H}_{2}{ }^{+}\left(v^{\prime}\right)+\mathrm{H}_{2}\left(v^{\prime \prime}\right) \rightarrow \mathrm{H}_{3}{ }^{+}\left(v^{\prime \prime \prime}\right)+\mathrm{H} \text {. }
$$

Finding an expression for $n_{\mathrm{H}_{2}}$ in terms of $n_{e}$ using quasineutrality, $n_{e}=n_{\mathrm{H}_{2}+}+n_{\mathrm{H}^{+}}$, we obtain

$$
n_{\mathrm{H}_{2}+}=\frac{n_{e} n_{\mathrm{H}_{2}(v \geqslant 4)} k_{\mathrm{cx}}}{n_{e} k_{\mathrm{dr}}+n_{\mathrm{H}_{2}(v \geqslant 4)} k_{\mathrm{cx}}+n_{\mathrm{H}_{2}}\left(k_{\mathrm{H}_{3}}+k_{\mathrm{cx}_{2}}\right)} .
$$

At low electron densities (for $n_{e}<10^{19} \mathrm{~m}^{-3}$ at $6.5 \mathrm{~Pa}$ ), $n_{e} k_{\mathrm{dr}}$ can be ignored and $n_{\mathrm{H}_{2}}$ becomes approximately proportional to $n_{e}$.

$$
\begin{aligned}
& \text { For } n_{e}>10^{19} \mathrm{~m}^{-3}, \\
& \qquad n_{\mathrm{H}_{2}+} \approx \frac{n_{\mathrm{H}_{2}(v \geqslant 4)} k_{\mathrm{cx}}}{k_{\mathrm{dr}}} .
\end{aligned}
$$

\section{B. Local population balance of $\boldsymbol{n}=\mathbf{2}$ excited atoms}

Excited neutrals in the $n=2$ excited state are assumed to be produced and destroyed locally, because within the radiative lifetime, these atoms can travel less than $0.1 \mathrm{~mm}$. Note that radiation transport can occur and effectively lead to transport of these neutrals. This effect is simulated in Sec. V.

The main production process of the $n=2$ state is MAR and relaxation from the $n=3$ state, as described above. The main destruction processes are electron (de-)excitation and radiation. Absorption of laser radiation and stimulated emission is neglected because the transition is not in saturation.

Assuming a steady-state plasma,

$$
\begin{aligned}
\frac{d n_{2}}{d t}= & 0=0.1 n_{\mathrm{H}_{2}+n_{e}} k_{\mathrm{dr}}+n_{3}\left(A_{32}+n_{e} k_{32}\right) \\
& -n_{2}\left(A_{21} \Lambda_{21}+n_{e} K_{2}\right) .
\end{aligned}
$$

$n_{j}$ is the density of the $n=j$ excited state, $A_{i j}$ is an Einstein coefficient, $\Lambda_{21}$ the escape factor for the Lyman- $\alpha$ line, $k_{i j}$ the rate of electron (de-)excitation from level $i$ to level $j$ (from Ref. [14]), and $K_{i}=\sum_{j} k_{i j}$ (from Ref. [15]). This yields

$$
n_{2}=\frac{0.1 n_{\mathrm{H}_{2}}+n_{e} k_{\mathrm{dr}}+n_{3}\left(A_{32}+n_{e} k_{32}\right)}{A_{21} \Lambda_{21}+n_{e} K_{2}} .
$$


A balance for the density of the $n=3$ excited state is obtained using the same procedure:

$$
n_{3}=\frac{0.45 n_{\mathrm{H}_{2}+} n_{e} k_{\mathrm{dr}}}{A_{3}+n_{e} K_{3}}
$$

where $A_{3}=\sum_{i} A_{3 i}$. Solving Eqs. (8) and (9) with $k_{32} / K_{3} \approx$ $A_{32} / A_{3} \approx 0.45$ gives, approximately,

$$
n_{2}=\frac{0.3 n_{\mathrm{H}_{2}+n_{e}} k_{\mathrm{dr}}}{A_{21} \Lambda_{21}+n_{e} K_{2}} .
$$

We distinguish the following regimes:

(a) For $n_{e}<10^{19} \mathrm{~m}^{-3}: n_{\mathrm{H}_{2}} \propto n_{e}$ and $A_{21} \Lambda_{21} \gg n_{e} K_{2}$, therefore

$$
n_{2} \propto n_{e}^{2} .
$$

(b) For $10^{19} \mathrm{~m}^{-3}<n_{e} \ll A_{21} \Lambda_{21} / K_{2}: n_{\mathrm{H}_{2}+} \approx n_{\mathrm{H}_{2}(v \geqslant 4)} k_{\mathrm{cx}} /$ $k_{\mathrm{dr}}$ and $A_{21} \Lambda_{21} \gg n_{e} K_{2}$, therefore

$$
n_{2} \approx \frac{0.3 n_{e} n_{\mathrm{H}_{2}(v \geqslant 4)} k_{\mathrm{cx}}}{A_{21} \Lambda_{21}} \text {. }
$$

(c) For $n_{e} \gg A_{21} \Lambda_{21} / K_{2}: \quad n_{\mathrm{H}_{2}+} \approx n_{\mathrm{H}_{2}(v \geqslant 4)} k_{\mathrm{cx}} / k_{\mathrm{dr}}$ and $A_{21} \Lambda_{21} \ll n_{e} K_{2}$, therefore

$$
n_{2} \approx \frac{0.3 n_{\mathrm{H}_{2}(v \geqslant 4)} k_{\mathrm{cx}}}{K_{2}} .
$$

These expressions give simple $n_{e}$ dependencies for $n_{2}$ in different electron-density ranges. However, the dependency of the escape factor of Lyman- $\alpha, \Lambda_{21}$ on $n_{e}$, is not yet considered. We consider the expected behavior of the escape factor $\Lambda_{21}$ below.

\section{Influence of the escape factor in the population balance}

The plasma becomes optically thick for Lyman- $\alpha$ (and to a lesser extent also for Lyman- $\beta$ ) if the ground-state neutral density, $n_{\mathrm{H}}$, in the plasma is sufficiently high. This density is expected to rise as a function of electron density in the plasma. However, the dependency is difficult to predict, especially at the measurement position, $4 \mathrm{~cm}$ from the plasma source. Ground-state atoms are long lived and can travel several centimeters in the axial direction before escaping radially from the plasma beam. The flux of $n_{\mathrm{H}}$ in the beam at the exit of the plasma source is expected to increase with source current, because the electron density inside the source channel increases [16] and the mean free path of these neutrals decreases. Local production of $n_{\mathrm{H}}$ in the beam outside the plasma source is expected to increase linearly with electron density because the rates of its two main production mechanisms, MAR and electron dissociation of molecules [13], are both proportional to $n_{e}$.

The dependency of the ground-state density near the plasma source will be estimated in this paper using the escape factor, $\Lambda_{21}$. For the low electron-density regime described in Eq. (12), the electron-density dependency of the $n=2$ density is $\mathrm{H}_{2}(v \geqslant 4)\left(n_{e}\right) n_{e} / \Lambda_{21}\left(n_{e}\right)$. Using measurements of the $n=2$ density vs $n_{e}$ and calculations of $\Lambda_{21}$ vs $n_{\mathrm{H}}$, we will obtain combined information about $n_{\mathrm{H}}$ and $n_{\mathrm{H}_{2}(v \geqslant 4)}$. The escape factor is taken from Behringer and Fantz [17], calculated for a cylindrical hydrogen plasma with a parabolic emission profile and a purely Gaussian-broadened spectral line. According to Rosado [18], the behavior of the escape factor for a Voigt spectral line shows almost identical behavior, down to an escape factor of less than 0.1. For an optical thickness, $k r$, of less than $1, \Lambda \approx 1 / k r \propto 1 / n_{\mathrm{H}}$.

The other important role of the escape factor in the $n=2$ population balance is its influence on the relative importance of loss by radiation and electron deexcitation. If electron deexcitation dominates, the $n=2$ density as a function of the electron density will saturate [Eq. (13)]. A lower escape factor will cause this saturation to occur at a lower value of the electron density.

\section{Radial dependencies of the population balance}

The radial density profile of $n=2$ excited neutrals depends sensitively on the radial profiles of $n_{e}$ and $n_{\mathrm{H}_{2}(v \geqslant 4)}$. According to Eqs. (11)-(13), at low electron densities the $n=2$ profile is dependent on the electron density and at high electron densities it depends only on the $\mathrm{H}_{2}(v \geqslant 4)$ density. The latter profile becomes hollow when the electron density is so high that the mean free path (determined by charge exchange in combination with elastic collisions) becomes significantly smaller than the plasma diameter. In that case, the resulting $n=2$ profile is also hollow.

Radiation transport of Lyman- $\alpha$ photons also affects the radial profile of $n=2$ excited neutrals. The radial density profile is broadened by reabsorption if the plasma is optically thick for these photons. A simple simulation of this effect has been carried out and is reported in Sec. V.

\section{EXPERIMENT}

\section{A. Experimental setup}

Pilot-PSI $[19,20]$ is a linear plasma generator (see Fig. 1). Hydrogen plasma is produced by a cascaded arc source [16,21] and flows into a vacuum vessel (typically 2-50 Pa). An axially directed magnetic field can be applied with a strength of up to $1.6 \mathrm{~T}$. The beam is $\sim 1 \mathrm{~cm}$ wide, $0.5 \mathrm{~m}$ long, and impinges on a conducting target at floating potential. Absorption and LIF measurements are performed at $4 \mathrm{~cm}$ from the source. The electron density and temperature are measured at the same position with Thomson scattering [2,3]. Statistics are excellent because of the high electron density.

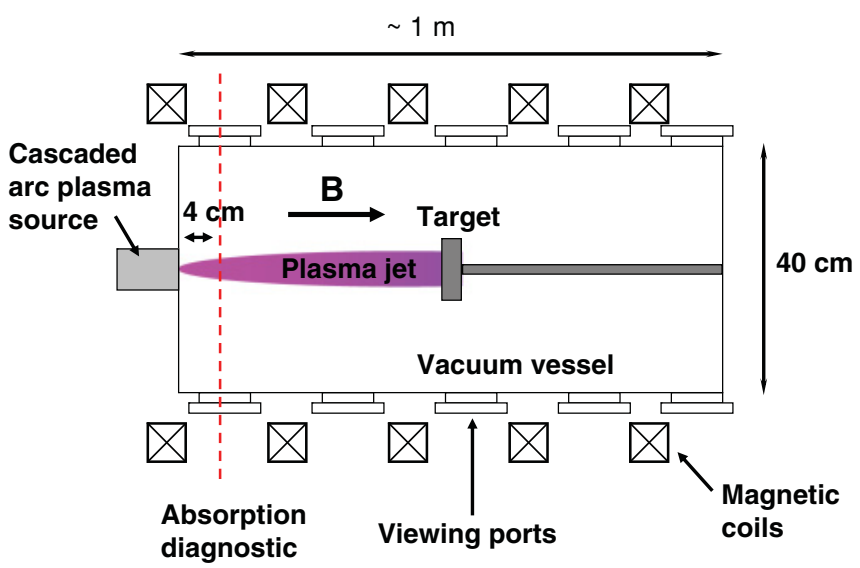

FIG. 1. (Color online) Plasma experiment Pilot-PSI. 


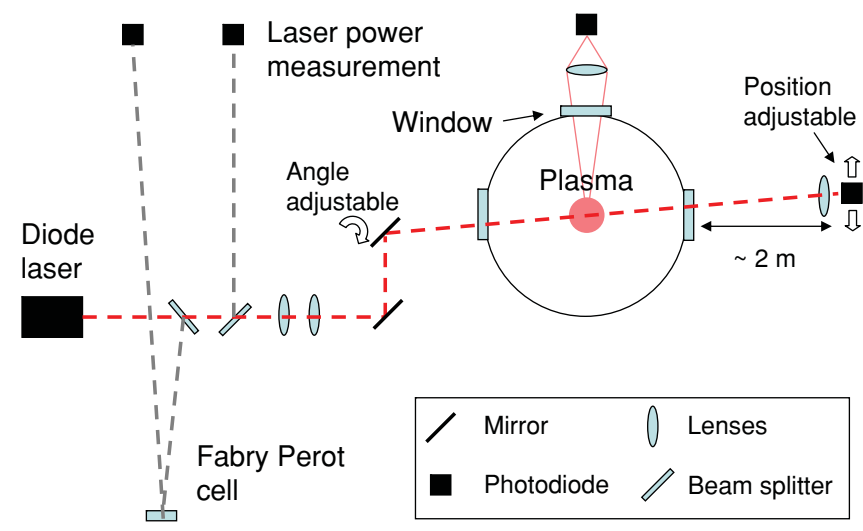

FIG. 2. (Color online) Diagnostic setup.

The optical setup for absorption and LIF is depicted in Fig. 2. Absorption is measured on the Balmer- $\alpha$ line from excited level $n=2$ to $n=3$. The LIF signal measured is the spontaneous radiative decay back to the $n=2$ level. We use a tunable external cavity diode laser (Vortex 6000, New Focus, wavelength range $656.33-656.60 \mathrm{~nm}$, horizontal polarization, spectral linewidth $<300 \mathrm{kHz}$ ). The laser power and wavelength are monitored in real time, the latter with a Fabry-Pérot cell. The laser is always at an angle to the windows to avoid interference effects. Scans as a function of electron density are mostly performed with an expanded beam (laser spot diameter up to $\phi=1 \mathrm{~cm}$ ) (with the aim of maximizing the fluorescence signal for LIF). Lateral scans are performed with $\phi<1.5 \mathrm{~mm}$.

For absorption, the laser is scanned across the radius of the plasma beam to obtain a lateral profile, by adjustment of the angle of the last mirror in front of the vessel. After a single pass through the plasma beam, the laser beam is focused onto a photodiode that measures the transmitted laser power. This photodiode is positioned at $1.5 \mathrm{~m}$ from the vessel to suppress background emission. The collection optics are adjusted during a radial scan, so that the laser beam is always focused onto the photodiode. The laser wavelength is scanned across the spectrum with a repetition frequency of $10 \mathrm{~Hz}$ with ten averages. Directly following each measurement, a calibration measurement is made with the magnetic field turned off. This reduces the laser absorption to $\ll 10^{-5}$.

Fluorescence is also collected onto a photodiode. Because the background emission is expected to be more than 1000 times stronger than the fluorescence, several techniques are employed to suppress it. First, lock-in detection is used with a mechanical chopper at $4 \mathrm{kHz}$, a spectrum scan at $0.05 \mathrm{~Hz}$, and a typical integration time of $2 \mathrm{~s}$. Also, both a Balmer- $\alpha$ filter and a slit are mounted in front of the photodiode such that only relevant light from regions where fluorescence is expected is imaged onto the photodiode.

\section{B. Overview of the plasma conditions}

The plasma conditions studied in the LIF and absorption experiments are characterized with Thomson scattering. These conditions are varied via the magnetic-field strength, the discharge current in the plasma source, and the background pressure in the vessel. A low electron-density range of $1-3 \times 10^{19} \mathrm{~m}^{-3}$ is achieved at $0.015 \mathrm{~T}$ and a high-density range of $2-10 \times 10^{20} \mathrm{~m}^{-3}$ at $0.4 \mathrm{~T}$.

Figure 3 gives an overview of representative electron density and temperature profiles and the change of peak values as a function of operation parameters. Figure 3(a) shows that the profiles are peaked and that the temperature profile is significantly wider than the density profile. In Fig. 3(b) the peak electron density is plotted as a function of discharge current. Interpolations are given for the exact pressures at which laser spectroscopy was performed. Figures 3(c) and

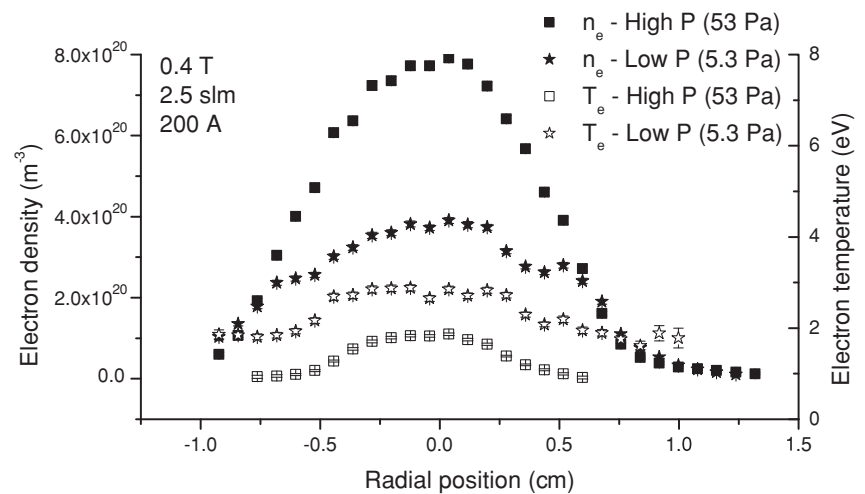

(a)

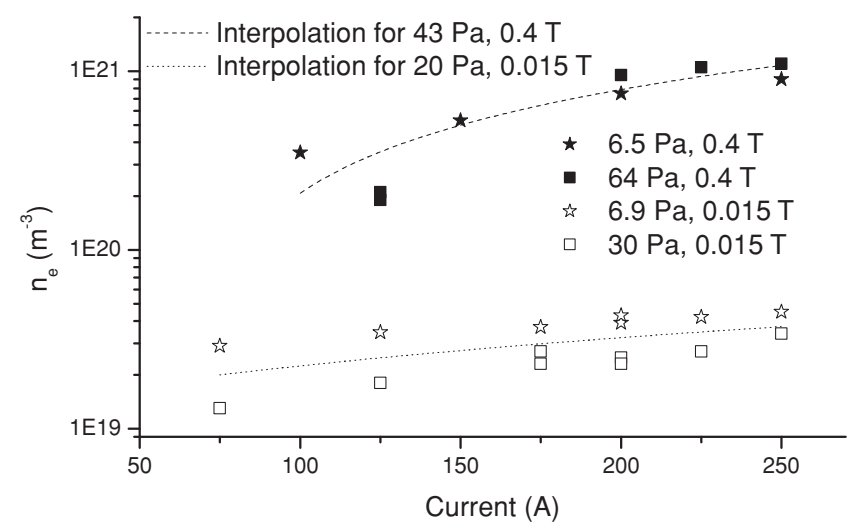

(b)

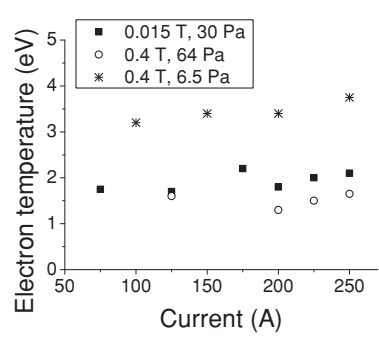

(c)

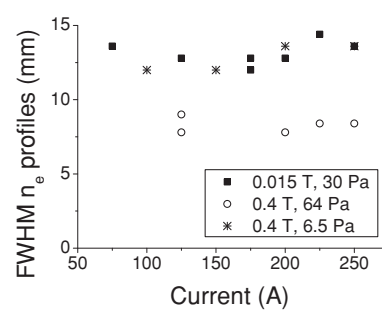

(d)
FIG. 3. Overview of plasma conditions studied in this paper. (a) Typical electron density and temperature profiles, corresponding to the lateral $n=2$ density measurements made at $0.4 \mathrm{~T}$. (b) Plasma conditions for electron density scans studied in this paper, characterized by peak electron density. Interpolations are for the background pressures in laser spectroscopy experiments. (c) Corresponding peak electron temperature and (d) full width half maximum (FWHM) of electron-profiles. Neither vary significantly as a function of current. 
3(d) show that the electron temperature and the plasma beam width are approximately independent of the discharge current.

\section{Analysis of data}

This section explains how $n=2$ densities are calculated from absorption and LIF signals. Also, the fitting procedure with which the spectra are analyzed in order to obtain ion velocity and temperature information is described.

\section{Absorption data analysis}

The $n=2$ density can be calculated from absorption on the nonsaturated Balmer- $\alpha$ transition by [22]

$$
n_{2}(r)=\frac{8 \pi}{\lambda_{0}^{2}} \frac{g_{2}}{g_{3}} \frac{\int k(\nu, r) d v}{A_{32}},
$$

where $\lambda_{0}$ is the central wavelength of the absorption spectrum, $g_{i}$ is the statistical weight of the $i$ th excited state, $k(v, r)$ is the absorption coefficient $\left(\mathrm{m}^{-1}\right)$, and $v$ is the frequency of the laser $(\mathrm{Hz})$. Note the units of $\int k(v) d v:\left(\mathrm{m}^{-1} \mathrm{~s}^{-1}\right)$.

The absorption measurements are line-of-sight integrals and measure the average of the quantity $k(v, r) l$ across the beam, where $l$ is the path length of the laser through the plasma. By spectrally integrating this data, we obtain results in the unit $\mathrm{s}^{-1}$, with which average $n=2$ densities are calculated with an assumed beam width.

Where lateral scans are performed, Abel inversion is used (as well as spectral integration) to obtain the quantity $\int k(v, r) d v$ (and subsequently the $n=2$ density) at local position $\mathrm{r}$.

\section{LIF data analysis}

When fluorescence on the (nonsaturated) Balmer- $\alpha$ transition is induced by absorption on the same transition, the $n=2$ density can be calculated from the measured LIF signal as follows. The amount of fluorescence photons emitted per cubic meter per second by the plasma is:

$$
Y_{\mathrm{LIF}}\left(\text { photons } \mathrm{m}^{-3} \mathrm{~s}^{-1}\right)=A_{32} n_{3}^{\mathrm{LIF}},
$$

where $n_{3}^{\mathrm{LIF}}$ is the density of $n=3$ excited neutrals that are created by absorption of laser photons. Its value is determined from a steady-state balance between its destruction and production processes:

$$
n_{3}^{\mathrm{LIF}}\left(A_{3}+n_{e} K_{3}+B_{32} \rho\right)=n_{2} B_{23} \rho .
$$

$\rho$ the laser power density $\left(\mathrm{J} \mathrm{s} \mathrm{m}^{-3}\right)$ and $B_{i k}$ is an Einstein coefficient. The signal is measured in $\mathrm{W} / \mathrm{nm}$, with a resolution in the order of $10 \mathrm{pm}$ (from Stark broadening). Integrating the signal over the spectrum gives us the total measurement signal $S_{\mathrm{LIF}}$ in watts. This is equal to

$$
S_{\mathrm{LIF}}=Y_{\mathrm{LIF}} \frac{\Omega}{4 \pi} T V_{\mathrm{det}} \frac{h c}{\lambda} .
$$

$\Omega$ is the volume angle of the detection system $\left(3 \times 10^{-2} \mathrm{sr}\right)$, $T$ is the transmission of the optics (estimated at 0.8), and $V_{\text {det }}$ is the detection volume. Using expressions (15)-(17), we can calculate the density of $n=2$ atoms from

$$
n_{2}=\frac{S_{\mathrm{LIF}}\left(A_{3}+n_{e} K_{3}+B_{32} \rho\right)}{(\Omega / 4 \pi) T V_{\mathrm{det}}(h c / \lambda) A_{32} B_{23} \rho} .
$$

\section{Spectral analysis}

Spectra are analyzed with a Voigt function [23] because Doppler and Stark broadening have comparable importance in the plasma considered. Asymmetric spectra are analyzed with a double Voigt function (detailed in Ref. [24]). The asymmetry is attributed to the existence of two distributions of $n=2$ excited neutrals, one coupled to the ions (hotter and rotating) and one not coupled to the ions (colder and not rotating). Ion properties can therefore be determined from (the "hot" component of) asymmetric spectra, and temperature from the Doppler width and velocity from the Doppler shift. The fine structure of $\mathrm{H} \alpha$ is simulated by splitting each Voigt function into two Voigt peaks shifted with respect to one another by $13 \mathrm{pm}$. One is 1.5 times higher than the other.

\section{LIF AND ABSORPTION MEASUREMENTS}

\section{A. Measurement data}

Absorption spectra are measured for two regimes: low electron density, at a magnetic-field strength of $0.015 \mathrm{~T}$, and high electron density at $0.4 \mathrm{~T}$. LIF could only be detected in the low electron-density regime. In the high electron-density regime, absorption measurements are made at high and low background pressure.

First we present the spectra measured in scans of the discharge current at different background pressures and magnetic-field strengths (see Fig. 4). They are made for the following three combinations of magnetic-field strength and background pressure: $0.015 \mathrm{~T}, 20 \mathrm{~Pa} ; 0.4 \mathrm{~T}, 64 \mathrm{~Pa}$; and $0.4 \mathrm{~T}$, $6.5 \mathrm{~Pa}$. All spectra are measured at the center of the beam except in Fig. 4(d), where the position was optimized for maximum signal. Absorption down to $7 \times 10^{-4}$ is measured (at high pressure and signal-to-noise ratio of 50), limited not by the diagnostic, but by the lower discharge current limit of the plasma source. At low pressure the minimum signal-to-noise ratio is 15 . The LIF measurements have a much lower signal-to-noise ratio and require lock-in detection (signal detectable down to $10^{-4}$ ). Notable is that the spectra measured off-center [Fig. 4(d)] are asymmetric (see Sec. III C 3 for an explanation).

Figure 5(a) displays the tests for saturation. The absorption and LIF signal are plotted as a function of laser power from zero to full power. The transition is clearly not saturated. Fluctuations in the signal are attributed to instability of the plasma. The tests are done at conditions of minimal quenching (where saturation is most probable). Low collisional deexcitation is ensured by low electron density at low magnetic-field strength. A minimal escape of Lyman radiation is chosen via high ground-state neutral density, achieved at a high background pressure and high discharge current (see Sec. VIB 1).

Next we present lateral scans performed at $0.4 \mathrm{~T}$ at both high and low background pressures. Figure 5 shows lateral absorption profiles at a magnetic-field strength $0.4 \mathrm{~T}$ and background pressures 6.5 and $50 \mathrm{~Pa}$. The scans are made with a laser spot of diameter $<1.5 \mathrm{~mm}$. The profiles are fit with three Gauss functions, allowing Abel inversion because Gauss functions are invariant under this procedure [25].

Density profiles for $n=2$ neutrals calculated from the results of this Abel inversion are shown in Fig. 6. 


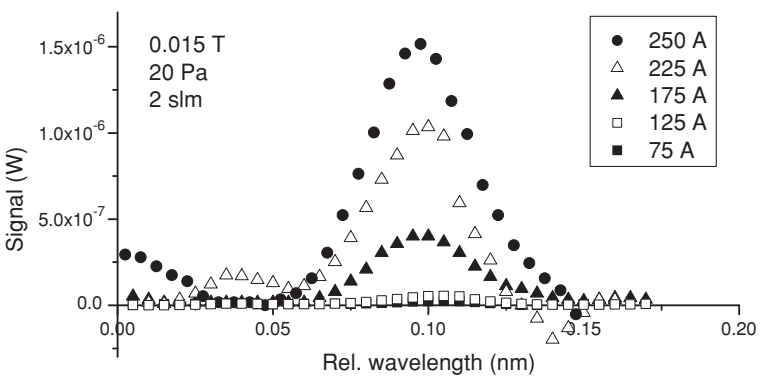

(a)

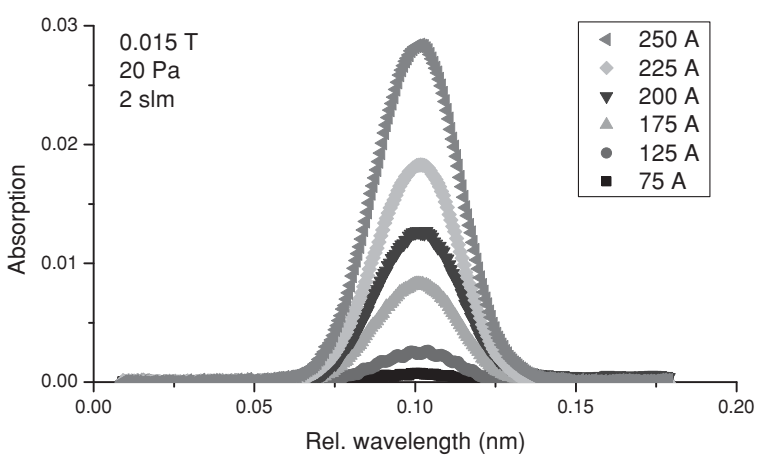

(b)

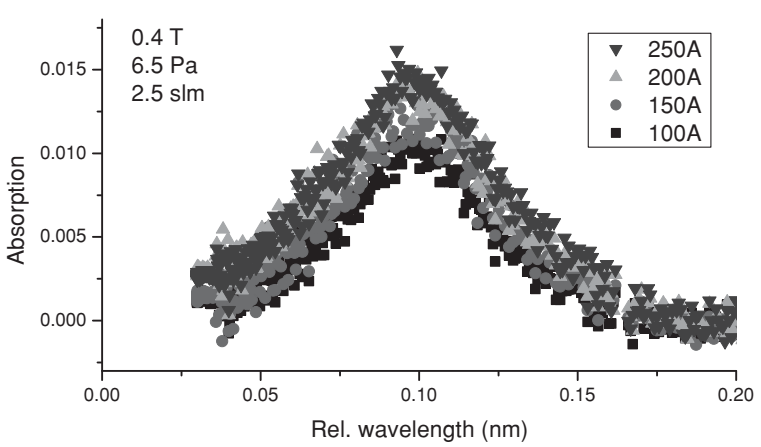

(c)

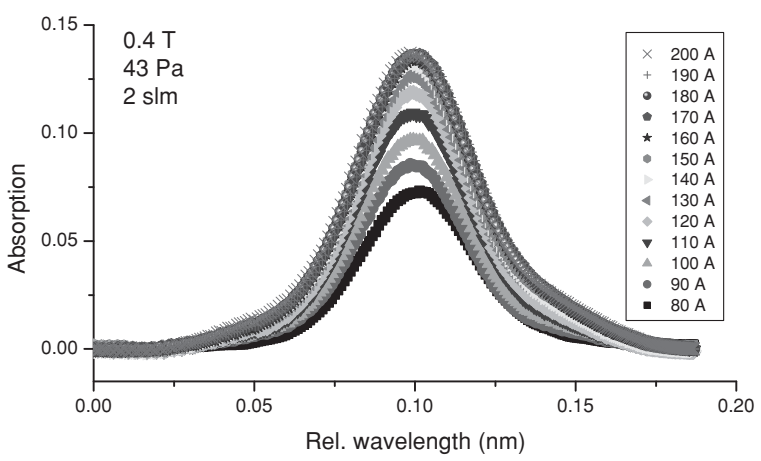

(d)

FIG. 4. Absorption and LIF spectra for varying source currents for three different plasma conditions. (a) LIF, low $n_{e}$, center of beam, $\phi_{\text {laser }} \approx 3 \mathrm{~mm}$. (b) Absorption, low $n_{e}$, center of beam, $\phi_{\text {laser }} \approx 3 \mathrm{~mm}$. (c) Absorption, high $n_{e}$, low $P$, center of beam, $\phi_{\text {laser }}<1.5 \mathrm{~mm}$. (d) Absorption, high $n_{e}$, high $P$, off-center, $\phi_{\text {laser }} \approx 1 \mathrm{~cm}$.

Electron-density profiles measured with Thomson scattering are also included for comparison.

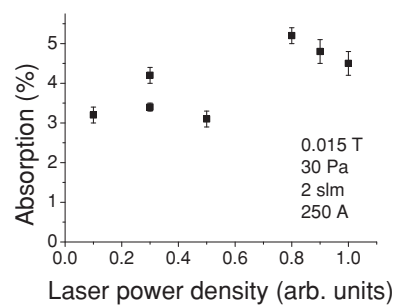

(a)

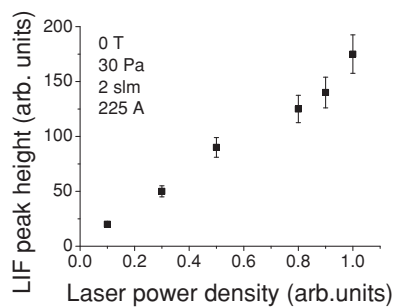

(b)

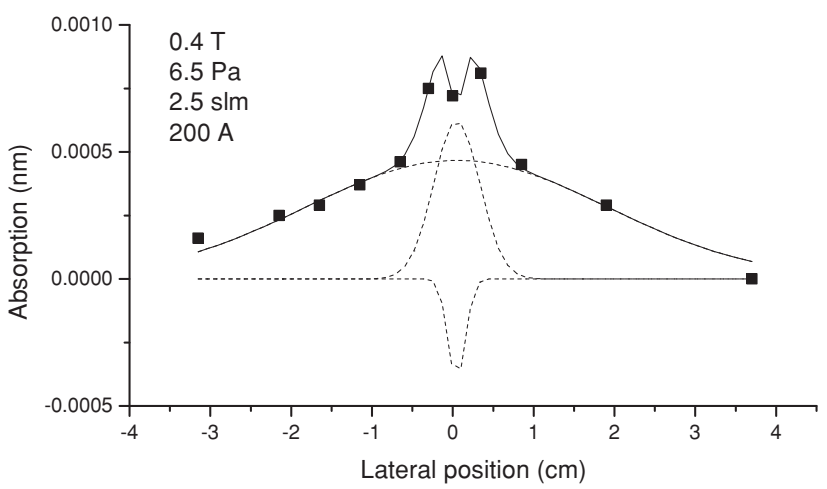

(c)

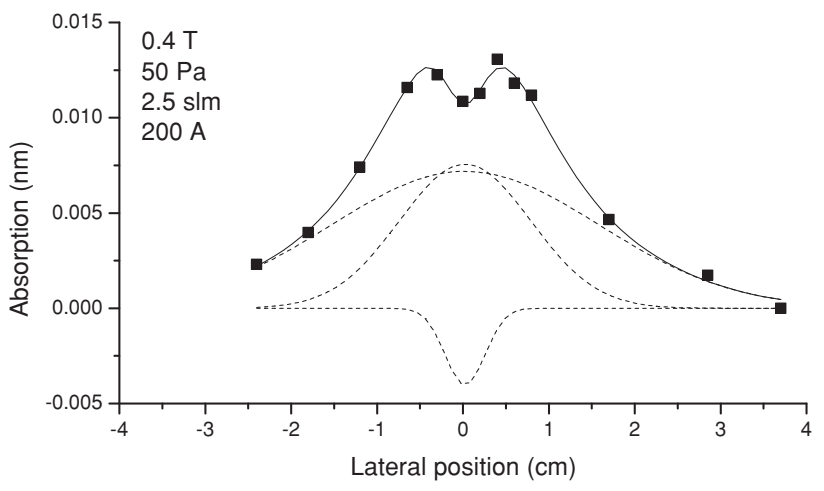

(d)

FIG. 5. Absorption in nanometers measured as a function of lateral distance at different background pressures, fitted with three Gaussian functions. (a) Absorption vs laser power density saturation test, quite unstable plasma. (b) LIF vs laser power saturation test. (c) $0.4 \mathrm{~T}$, low $P, \phi_{\text {laser }}<1.5 \mathrm{~mm}$. (d) $0.4 \mathrm{~T}$, high $P, \phi_{\text {laser }}<1.5 \mathrm{~mm}$.

The $n=2$ density profiles comprise three clear components: production inside the electron-density profile, production outside the electron-density profile by reabsorption of Lyman- $\alpha$, and a hollow region in the center of the beam (no production at all). For both measurements, the $n=2$ profiles become strikingly hollow for an electron density above $n_{e} \approx 4-5 \times 10^{20} \mathrm{~m}^{-3}$. For the $6.5 \mathrm{~Pa}$ measurement, the Abel inversion returns a negative density in the center of the beam, which probably indicates that the real profile is steeper than can be simulated with a Gauss function. To estimate the error made, we integrated the (positive parts of) the profile and compared it to the integrated density measured at the center of the beam. This gives a discrepancy of $1 \%$. The largest difference between the lateral scans at high and low pressure is the profile shape of the reabsorption production outside the plasma 


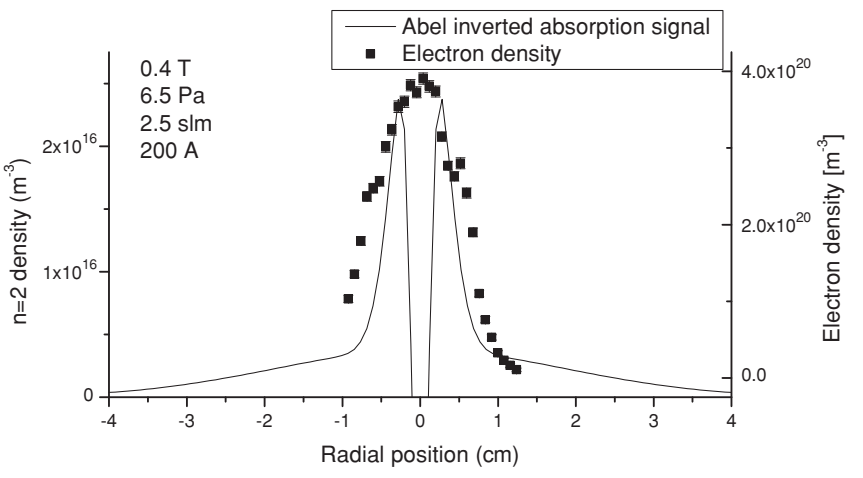

(a)

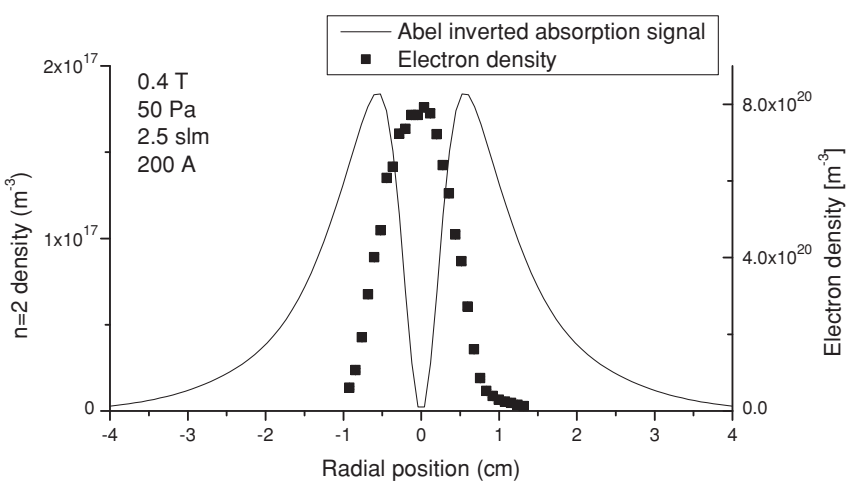

(b)

FIG. 6. $n=2$ density as a function of radius determined from Abel inversion of lateral absorption scans. Electron-density profiles are plotted for comparison. (a) $0.4 \mathrm{~T}$, low $P$. (b) $0.4 \mathrm{~T}$, high $P$.

beam. These profiles will be simulated and discussed further in Sec. V.

Third, we present $n=2$ densities calculated from the LIF and absorption spectra in Fig. 4 as a function of (central) electron density (see Fig. 7). As stated earlier, LIF could only be observed for the lowest electron-density condition: $B=0.015 \mathrm{~T}$ and $P=20 \mathrm{~Pa}$. Where the background pressures

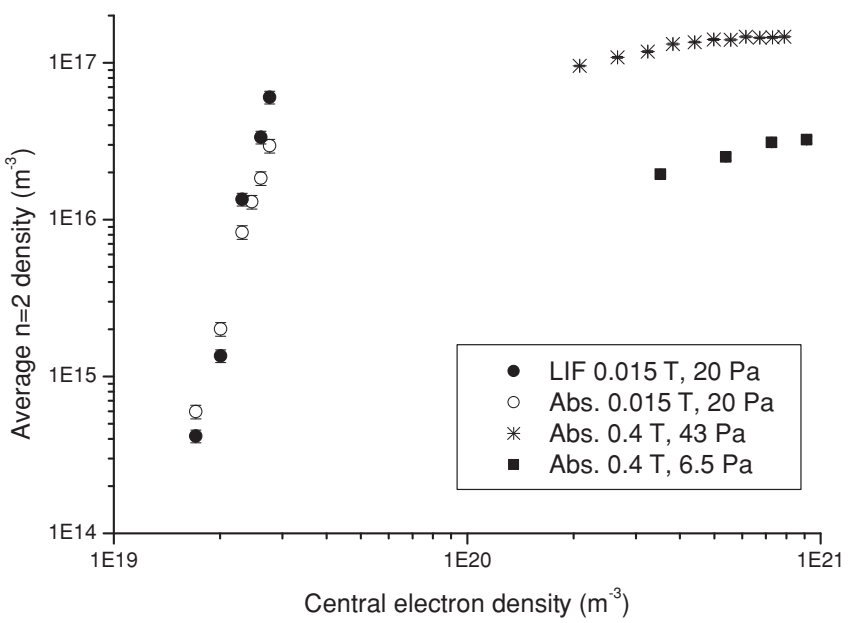

FIG. 7. Average $n=2$ densities from LIF and absorption as a function of the central electron density. Where necessary, electron densities are calculated by interpolation in Fig. 3(b). are different for $n=2$ density and Thomson scattering measurements, interpolation is performed to obtain the electron density, as shown in Fig. 3(b).

The densities from absorption spectra are averages over the diameter of the beam, whereas the densities from LIF spectra are averages over the central $3 \mathrm{~mm}$ of the beam (the laser spot diameter). In the calculation of the $n=2$ density from LIF, a detection volume $V_{\text {det }}=3.4 \mathrm{~cm}^{3}$ was chosen to obtain agreement with the absolute density determined from absorption. For the calculation of averages for absorption, the width of the $n=2$ density profile is assumed to be equal to the width of the electron-density profile [see Fig. 3(d)]. For the high-pressure case $(0.4 \mathrm{~T}, 43 \mathrm{~Pa})$ we make an exception. Here the $n=2$ profile is expected to be significantly wider than the electron-density profile, as evident from Fig. 6. We assume a width of $2 \mathrm{~cm}$. For the low magnetic-field case $(0.015 \mathrm{~T}, 20 \mathrm{~Pa})$, the beam width probably increases with current, as the reabsorption of Lyman- $\alpha$ owing to increased ground-state density (see Sec. VI B 1) increases. The maximum $n=2$ density (at $0.015 \mathrm{~T}, 250 \mathrm{~A}$ ) is therefore likely to be up to a factor of 2 lower than plotted.

\section{B. Spectral analysis}

Absorption spectra measured at high magnetic-field strength $(0.4 \mathrm{~T})$ are found to be asymmetric (similar to the asymmetry found in $\mathrm{H} \beta$ emission spectra in Ref. [24]). This asymmetry is attributed to the existence of two populations of the excited neutrals being probed. In Ref. [24], the hotter population was attributed to charge exchange of excited neutrals with plasma ions and the colder population to collisions of excited neutrals with colder background neutrals.

Given the importance of reabsorption of Lyman- $\alpha$ observed in Fig. 6, we now propose an additional mechanism for the existence of two $n=2$ density distributions. The hot, rotating distribution is created by MAR, the last step of which, dissociative recombination, involves hot $\mathrm{H}_{2}{ }^{+}$ions and electrons. The $\mathrm{H}_{2}{ }^{+}$ions are coupled to the $\mathrm{H}^{+}$ions because they undergo typically six collisions with these ions before dissociative recombination. The cold, nonrotating population is created by reabsorption. Lymann- $\alpha$ photons are reabsorbed by cold, nonrotating ground-state atoms. These atoms do not live long enough to undergo charge exchange before they radiate again.

Assuming that this is the correct explanation for the double $n=2$ density distribution, we can measure the proportion of $n=2$ excited neutrals in the beam that are created by dissociative recombination and reabsorption respectively. We simply fit the spectra with a double Voigt function and compare the areas under the Voigt functions corresponding to the "hot" and the "cold" neutral populations. This ratio is $\sim 2 / 1$ for $P=6.5 \mathrm{~Pa}$ and $0.4 \mathrm{~T}$ and $\sim 2 / 3$ at the same conditions but a higher pressure of $50 \mathrm{~Pa}$.

Ion temperature and velocity determinations are not affected by this additional explanation of the double distribution. These parameters are determined for the asymmetric spectra measured at high magnetic field $(0.4 \mathrm{~T})$, and plotted as a function of lateral position in Figs. 8 and 9, for high and low pressure, respectively. The symmetric 


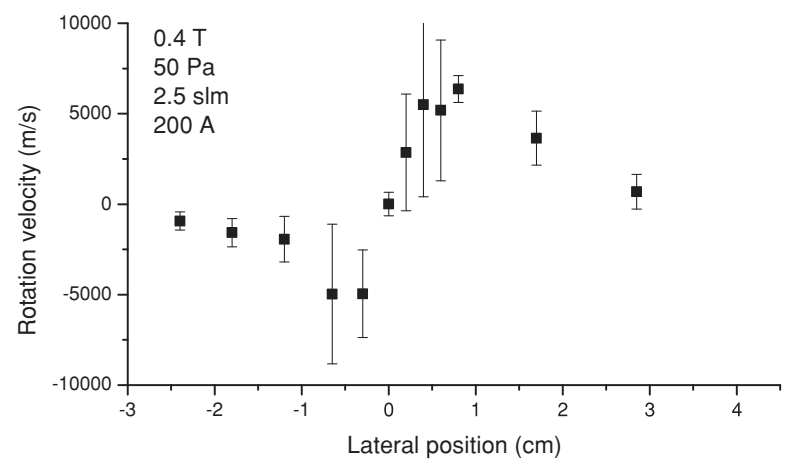

(a)

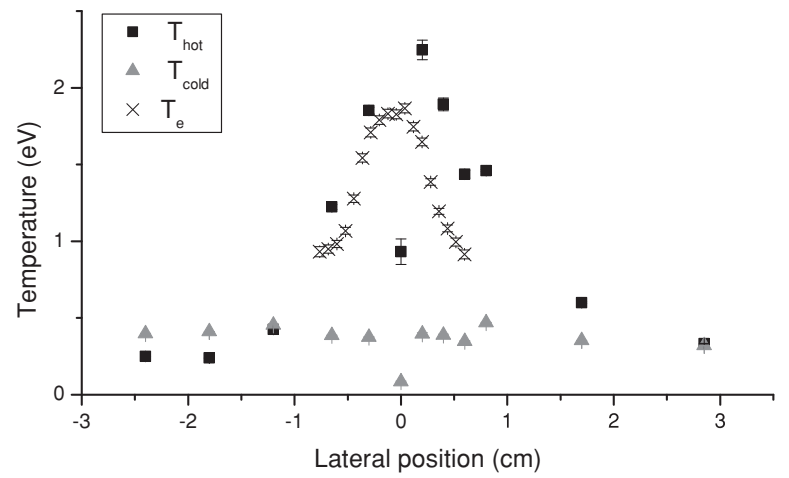

(b)

FIG. 8. (a) Rotation velocities and (b) temperatures deduced from double Voigt fits of spectra measured in a lateral absorption scan of the plasma beam at $50 \mathrm{~Pa}$ and $0.4 \mathrm{~T}$.

spectra outside of the plasma beam are fit with a Gaussian function (accounting for fine splitting as explained in Sec. III C 3). The maximum rotation velocity is $5-7 \mathrm{~km} / \mathrm{s}$ in both cases. At lower pressure, the errors become larger. This is owing to the lower density of $n=2$ and could be solved by improving the sensitivity of the diagnostic (see Sec. VII). The "hot" and "cold" neutral temperatures are plotted and compared to the electron temperature. The results for the temperatures of the $n=2$ distributions are reasonably systematic. One notable exception is the central point at high pressure. Here, the Stark width seems to have been overestimated and the Doppler width underestimated in the fitting procedure. The temperature of the "hot" component is in reasonable agreement with the electron temperature. We should, however, remember that the spectra are line-of-sight measurements. Determined temperatures are averages over a chord of the plasma beam and are thus expected to be underestimates of the local, central ion temperature. As in Ref. [24], ion viscous heating is suggested as a cause for a hotter ion than electron temperature.

At low magnetic-field strength $(0.015 \mathrm{~T})$, the spectra are symmetric and are fit with a single Voigt function; ion properties cannot be determined. Here, the temperature of the $n=2$ population is typically $0.3 \mathrm{eV}$, while the electron temperature is $\sim 2 \mathrm{eV}$. The average of the whole $n=2$ population is poorly coupled to the ions.

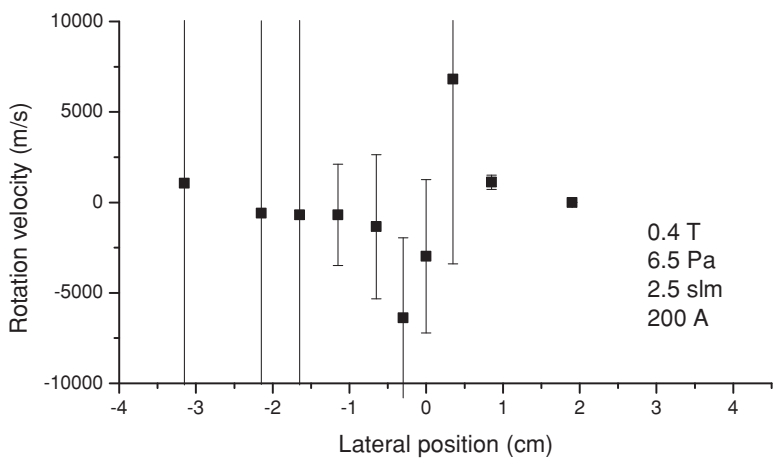

(a)

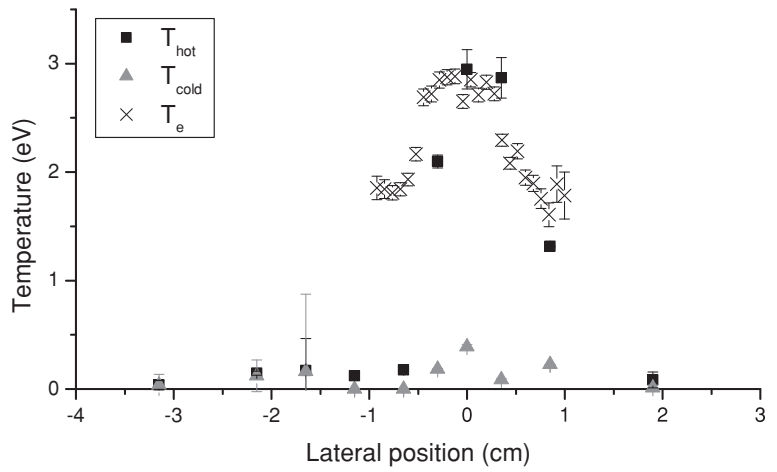

(b)

FIG. 9. (a) Rotation velocities and (b) temperatures deduced from double Voigt fits of spectra measured in a lateral absorption scan of the plasma beam at $50 \mathrm{~Pa}$ and $0.4 \mathrm{~T}$.

\section{MONTE CARLO SIMULATION OF THE RADIATION TRANSPORT}

By simulating the $n=2$ production by reabsorption of Lyman- $\alpha$ photons and comparing this to the measurement profiles (Fig. 6), we are able to estimate the density of ground-state neutrals just outside the plasma beam. These $n=2$ atoms could not be produced in any other way. MAR only takes place in the presence of electrons (in the plasma beam) and $n=2$ neutrals can only travel $0.1 \mathrm{~mm}$ or less within their lifetime.

\section{A. Method}

Radiation transport in the plasma beam of Pilot-PSI was simulated in a Monte Carlo code by following a Lyman- $\alpha$ photon emitted in the plasma beam as it is continually emitted and reabsorbed until it hits the vessel wall at a radius of $20 \mathrm{~cm}$. The simulation assumes a "hot" population of $n=2$ excited atoms produced by MAR and a "cold" population produced by reabsorption of Lyman- $\alpha$ photons, as described in Sec. III C 3. At the start of the simulation, the photon is given a random frequency, with probabilities corresponding to a Doppler profile consistent with the measured temperature of the ("hot" population of) $n=2$ excited particles. After each subsequent reabsorption, a new random frequency is chosen for the photon from the expected Doppler spectral profile of colder ground-state atoms. The number of reabsorptions is recorded in each volume $V=2 \pi r d r d z$. This calculation is repeated 10000 times and the result is averaged. 
The rate of reabsorption in a certain volume $V$ is equal to the amount of reabsorptions, $N_{a}$, in that volume owing to one photon, multiplied by the number of "primary" photons produced per second in the plasma in slice $d z$. The rate of production of "primary" photons is the number of "primary" ("hot") $n=2$ atoms in the plasma beam in $d z$ multiplied by the Einstein coefficient, $A_{21}$. We estimate the number of "hot" $n=2$ excited neutrals as the density, $n_{2}^{\text {hot }}$, multiplied by the approximate volume in which they are created, $V$.

The plasma is at steady state, so we can set the rate of reabsorption of Lyman- $\alpha$ equal to the rate of destruction of "secondary" $n=2$ neutrals:

$$
N_{a}(r) n_{2}^{\text {hot }} V \alpha A_{21}=n_{2}(r)\left(A_{21}+n_{e} K_{2}\right) .
$$

So,

$$
n_{2}(r)=\frac{A_{21}}{A_{21}+n_{e} K_{2}} N_{a}(r) n_{2}^{\text {hot }} V \alpha
$$

The radiation transport calculation was carried out for ground-state atoms $n_{\mathrm{H}}$ with a range of densities and temperatures. It was found that the $n=2$ density profile resulting from reabsorption was much less sensitive to the temperature of $n_{\mathrm{H}}$ than to its density. For the results presented in this paper, the temperature of ground-state atoms was assumed to be $1000 \mathrm{~K}$.

Our aim was to simulate as closely as possible the shape of the lateral absorption profiles shown in Fig. 6 measured at 6.5 and $50 \mathrm{~Pa}$ in order to make an estimation of the density of $n_{\mathrm{H}}$ inside and outside of the beam. The tactic used was to assume a flat radial profile of $n_{\mathrm{H}}$ and to try different values of $n_{\mathrm{H}}$ such that the measured $n=2$ density was matched as closely as possible. The measured number of 'hot $n=2$ excited atoms in the plasma was estimated by approximating the average $n=2$ density to be $2 / 3$ of the peak value and taking the volume to be the region in which the electron density is between 1.5 and $3.5 \times 10^{20} \mathrm{~m}^{-3}$ (above this electron-density range the peak becomes hollow and below this range the production is negligible). This resulting number was multiplied by the fraction of neutrals that are 'hot as deduced from the double Voigt fits (see Sec. IV B). This ratio was $2 / 3$ for the $6.5 \mathrm{~Pa}$ measurement and $2 / 5$ for the $50 \mathrm{~Pa}$ measurement. The values used in the simulation are $n_{2}^{\text {hot }}=4.8 \times 10^{16} \mathrm{~m}^{-3}$, $V=5.89 \times 10^{-5} \mathrm{~m}^{-3}, T_{\text {hot }}=2.0 \mathrm{eV}, T_{\text {cold }}=1000 \mathrm{~K}$ for $P=$ $6.5 \mathrm{~Pa}$ and $n_{2}^{\text {hot }}=1.044 \times 10^{16} \mathrm{~m}^{-3}, V=1.34 \times 10^{-4} \mathrm{~m}^{3}$, $T_{\text {hot }}=2.5 \mathrm{eV}, T_{\text {cold }}=1000 \mathrm{~K}$ for $P=50 \mathrm{~Pa}$.

\section{B. Results}

We simulated the $n=2$ lateral density scan measurements (Fig. 6) made at 6.5 and $50 \mathrm{~Pa}$. The results are shown in Fig. 10. The measured value of the density of $n=2$ neutrals is plotted at five positions across the radius. This is the total $n=2$ density, including both the hot and the cold population. The expected density of the cold population inside the beam (based on measurements of the ratio between the hot and cold population) is indicated by a star. Outside the beam, all measured $n=2$ atoms are expected to be cold. The calculated radial $n=2$ density distribution is plotted at different assumed values of the ground-state density, $n_{\mathrm{H}}$. As explained above, the radial profile of $n_{\mathrm{H}}$ is assumed

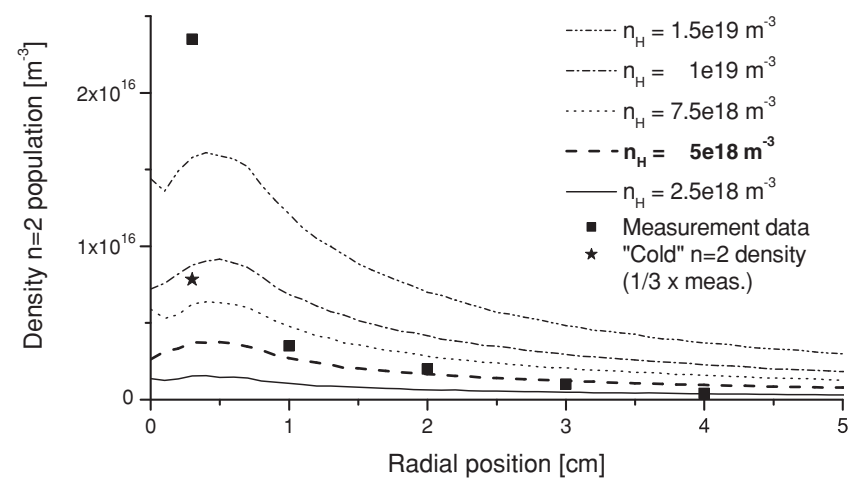

(a) $\mathrm{P}=6.5 \mathrm{~Pa}$

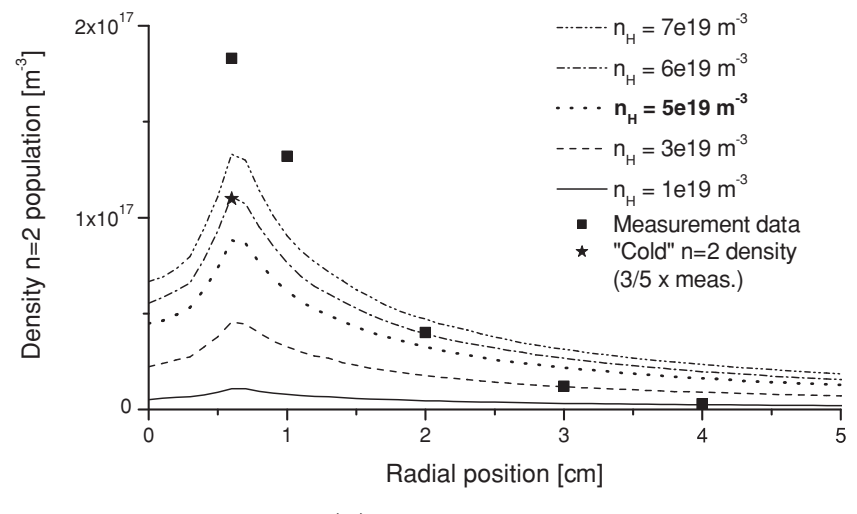

(b) $\mathrm{P}=50 \mathrm{~Pa}$

FIG. 10. Simulation of $n=2$ production owing to radiation transport with different values of ground-state density, $n_{\mathrm{H}}$. Measurement data points are added for comparison.

to be flat. It is evident that the measurements show a faster drop in density than the simulation. This probably indicates a radial gradient in $n_{\mathrm{H}}$, but could also be partly owing to the inaccuracy of the Abel inverted profile. A radial gradient is, however, to be expected in $n_{\mathrm{H}}$, because hydrogen atoms, even in the ground state, are confined by charge exchange reactions with ions when the ion density is higher than $\sim 10^{20} \mathrm{~m}^{-3}$. Outside the beam, the ground-state neutral density is expected to decrease radially owing to diffusion.

The best overall match between measured and simulated "cold" $n=2$ atoms is found at approximately $n_{\mathrm{H}}=$ $5 \times 10^{18} \mathrm{~m}^{-3}$ for $6.5 \mathrm{~Pa}$ and $n_{\mathrm{H}}=5 \times 10^{19} \mathrm{~m}^{-3}$ for $50 \mathrm{~Pa}$.

These solutions give an underestimation of the $n=2$ density in the center of the beam and an overestimation at the edge. We conclude that the value of the neutral density in the center is higher than determined here. This would lead to more buildup of the $n=2$ density in the center of the beam, and give a steeper radial $n=2$ density profile than simulated (more reabsorptions and thus more photon loss). This would give a better match of the $n=2$ density at the edge for the estimations of the ground-state density made above. A gradient would be required in the assumed $n_{\mathrm{H}}$ density in the simulation to achieve more accurate results. This was not performed here owing to insufficient accuracy of the Abel inversions. 


\section{INTERPRETATION OF THE RESULTS}

\section{A. Estimation of the escape factor from spectral analysis}

The escape factor of Lyman- $\alpha$ is important to know for collisional-radiative modeling of the plasma of Pilot-PSI. If this line is optically thick, then both the excitation and ionization balance will lean toward higher excitation and/or ionization than in the optically thin case.

An estimate can be made of the Lyman- $\alpha$ escape factor $\Lambda_{21}$ by assuming as hypothesized in Sec. III C 3 that there is both a 'hot and a "cold distribution of $n=2$ atoms in the plasma populated respectively by MAR and reabsorption of Lyman- $\alpha$ photons. We illustrate as follows.

Considering a case in which Lyman- $\alpha$ is optically thin and all $n=2$ neutrals are produced by MAR and are 'hot, the population balance for the $n=2$ density, $n_{2}^{\text {thin }}$, can be written as

$$
n_{2}^{\text {thin }}=n_{2}^{\text {hot }}=\frac{0.3 n_{e} n_{\mathrm{H}_{2}(v \geqslant 4)} k_{\mathrm{cx}}}{A_{21}+n_{e} K_{2}} .
$$

By then considering a plasma in which Lyman- $\alpha$ is optically thick, we obtain

$$
\begin{aligned}
n_{2}^{\text {total }} & =\frac{0.3 n_{e} n_{\mathrm{H}_{2}(v \geqslant 4)} k_{\mathrm{cx}}}{A_{21} \Lambda_{21}+n_{e} K_{2}} \\
& =n_{2}^{\text {hot }} \cdot \frac{A_{21}+n_{e} K_{2}}{A_{21} \Lambda_{21}+n_{e} K_{2}} \\
& =\frac{n_{2}^{\text {hot }}}{\alpha},
\end{aligned}
$$

where

$$
\begin{aligned}
\alpha & =\frac{n_{2}^{\text {hot }}}{n_{2}^{\text {total }}} \\
& =\frac{A_{21} \Lambda_{21}+n_{e} K_{2}}{A_{21}+n_{e} K_{2}} .
\end{aligned}
$$

If we know $\alpha$, the ratio of $n=2$ neutrals that are hot, we can then calculate the escape factor $\Lambda_{21}$ from

$$
\Lambda_{21}=\frac{\alpha\left(n_{e} K_{2}+A_{21}\right)-n_{e} K_{2}}{A_{21}} .
$$

Using an average lateral value for the electron density of $2 \times 10^{20} \mathrm{~m}^{-3}$ at $6.5 \mathrm{~Pa}$ and $4 \times 10^{20} \mathrm{~m}^{-3}$ at $50 \mathrm{~Pa}$, and taking the hot-to-cold ratios as $2 / 3$ and $2 / 5$, respectively, we calculate that $\Lambda_{21}=0.57$ at $6.5 \mathrm{~Pa}$ and $\Lambda_{21}=0.145$ at $50 \mathrm{~Pa}$. Using the escape factor simulation in Ref. [17], this gives neutral densities of $2.22 \times 10^{19} \mathrm{~m}^{-3}$ and $1.11 \times 10^{20} \mathrm{~m}^{-3}$, respectively. Taking the electron density in the center of the beam, this corresponds to ionization degrees of $95 \%$ and $88 \%$, respectively.

In both cases the Lyman- $\alpha$ radiation field will have a significant effect on the excitation balance in the plasma, more so at high pressure than at low pressure.

\section{B. Examination of the $\boldsymbol{n}=\mathbf{2}$ density behavior for determination of neutral densities}

In Sec. II, we ascertained that electrons, rovibrationally excited molecules, and ground-state neutrals all play an important role in the excitation balance of $n=2$ excited neutrals. In order to deduce density information about the neutral populations mentioned above, inside and outside of the plasma beam and at different electron densities, we now analyze the $n=2$ measurements made as a function of electron density (Fig. 7) and as a function of lateral distance (Fig. 6). A summary of the different neutral population densities determined is given in Sec. VIC.

\section{Density of ground-state atoms and rovibrationally excited molecules in the transition to the high electron-density regime}

In this section we attempt to understand how $n_{\mathrm{H}}$ and $n_{\mathrm{H}_{2}(v \geqslant 4)}$ change in Pilot-PSI in the transition to the high electron densities. We look at the $n=2$ density measurements as a function of electron density at low magnetic-field strength $(0.015 \mathrm{~T})$ in Fig. 7 . The rise in $n=2$ density is much steeper than the linear increase predicted by Eq. (12). An explanation could be that either the $\mathrm{H}_{2}(v \geqslant 4)$ density or $\Lambda_{21}$ or (most probably) both exhibits an $n_{e}$ dependence. We consider other possible contribution to the rise to be unimportant. The electron temperature remains approximately constant, ruling out a change in the production rate of $n=2$ excited atoms. A change in the width of the beam owing to reabsorption would at very most explain a factor of 2 in the $n=2$ density rise.

To explain the data, the factor $n_{\mathrm{H}_{2}(v \geqslant 4)} / \Lambda_{21}$ must rise by a factor of 30 from the lowest to the highest current value. We can estimate the contribution of $n_{\mathrm{H}_{2}(v \geqslant 4)}$ to this rise by noting the restriction on $\Lambda_{21}$. We estimated its value to be 0.145 at conditions conducive to high optical thickness (high magnetic field and high background pressure), so $0.145<\Lambda_{21}<1$. This gives $\mathrm{H}_{2}(v \geqslant 4)<2 \times 10^{19} \mathrm{~m}^{-3}$ at the lowest source current and $n_{\mathrm{H}_{2}(v \geqslant 4)}>8 \times 10^{19} \mathrm{~m}^{-3}$ at the highest source current. The minimum rise in the percentage of molecules that have an internal energy of $2 \mathrm{eV}$ is from $4.3 \%$ to $19.4 \%$. This is a large rise, especially if we compare it to $0.14 \%$ from other measurements [26] in a similar machine at $0 \mathrm{~T}$ and low electron density $\left(<10^{20} \mathrm{~m}^{-3}\right)$. A rise in $\Lambda_{21}$ from 0.145 to 1 corresponds to a rise in the ground-state atomic density from $n_{\mathrm{H}}<2.2 \times$ $10^{18} \mathrm{~m}^{-3}$ to $n_{\mathrm{H}}=1.1 \times 10^{20} \mathrm{~m}^{-3}$, which is much faster than the rise in electron density.

A faster rise in the ground-state atomic density than in the electron density is plausible, because $n_{\mathrm{H}}$ is not simply a direct function of the electron density. Ground-state atoms are not only produced locally, but can travel several centimeters along the axis before they exit the beam. Furthermore, the flux of neutrals exiting the beam is expected to increase with source current, because as the electron density inside the source increases with current [16], the atoms will be more and more confined by charge-exchange reactions. The density of rovibrationally excited molecules can be expected to increase with atomic density as its rate of production at the wall [26] will increase.

\section{Examination of lateral absorption profiles-determination of the ionization degree and the $\mathrm{H}_{2}(v \geqslant 4)$ density}

It is interesting to note that in Fig. 6 the lateral $n=2$ density profiles both become hollow at an electron density of $4-5 \times 10^{20} \mathrm{~m}^{-3}$. This agrees well with the values of electron density at which saturation is observed in the $n=2$ density in Fig. 7, and can be explained by the radial profile of rovibrationally excited molecules that becomes hollow at high 
electron density. The mean free path of these molecules is equal to the radius of the beam $(\sim 6 \mathrm{~mm})$ for $n_{e}=4 \times 10^{20} \mathrm{~m}^{-3}$ (molecular temperature: $\sim 0.3 \mathrm{eV}$, MAR rate coefficient: $3 \times 10^{-15} \mathrm{~m}^{3} \mathrm{~s}^{-1}$ [12]) and grows shorter as the electron density increases. Taking into account elastic collisions, the mean free path of these molecules will be even shorter.

The hollow $n=2$ excited neutral profiles yield the useful information that there is very little $n=2$ production in the center of the beam. Knowing the electron excitation rate of ground-state atoms to the $n=2$ level, we can therefore estimate an upper limit on the density of ground-state hydrogen in the center of the beam. The Abel inversion of the lateral $n=2$ density measurement tells us that there is zero $n=2$ density in the center of the beam. Allowing for the uncertainty in the Abel inversion (with a wide margin), we can assume that the $n=2$ density in the center has a maximum possible value of half of the peak value. To calculate a maximum ground-state density $n_{\mathrm{H}}$, we assume that there are no other production mechanisms apart from electron excitation from the ground state that contribute to the $n=2$ density in the center of the beam. We also assume maximum efficiency of the loss mechanisms and therefore that the escape factor $\Lambda_{21}$ is equal to 1 . This gives us the balance

$$
n_{\mathrm{H}}=\frac{n_{2}\left(n_{e} K_{2}+A_{21} \Lambda_{21}\right)}{n_{e} K_{12}} .
$$

For the 6.5 Pa measurement, we assume a maximum $n=2$ density in the center of the beam of $1.2 \times 10^{16} \mathrm{~m}^{-3}$. This gives us a maximum possible ground-state neutral density in the very center of the beam of $n_{\mathrm{H}}=7 \times 10^{19} \mathrm{~m}^{-3}$. Given the central electron density of $4 \times 10^{20} \mathrm{~m}^{-3}$, we obtain a minimum ionization degree in the center of the beam of $85 \%$. For the $50 \mathrm{~Pa}$ measurement we assume a maximum $n=2$ density of $9 \times 10^{16} \mathrm{~m}^{-3}$ and obtain a maximum $n_{\mathrm{H}}$ density of $3.7 \times$ $10^{20} \mathrm{~m}^{-3}$ and a minimum ionization degree of $68 \%$.

Another important feature of the lateral $n=2$ density measurements is the absolute value of the peak $n=2$ density. We can use it to estimate the density of $\mathrm{H}_{2}(v \geqslant 4)$ in the vessel by setting up a balance between creation and destruction of $n=2$ neutrals:

$$
\begin{gathered}
n_{2}\left(n_{e} K_{2}+\Lambda_{21} A_{21}\right)=0.3 n_{e} n_{\mathrm{H}_{2}(v \geqslant 4)} k_{\mathrm{cx}}, \\
n_{\mathrm{H}_{2}(v \geqslant 4)}=\frac{n_{2}\left(n_{e} K_{2}+\Lambda_{21} A_{21}\right)}{0.3 n_{e} k_{\mathrm{cx}}} .
\end{gathered}
$$

For $P=6.5 \mathrm{~Pa}$, this gives $n_{\mathrm{H}_{2}(v \geqslant 4)}=3.9 \times 10^{19} \mathrm{~m}^{-3}$, and for $P=50 \mathrm{~Pa}$, this gives $n_{\mathrm{H}_{2}(v \geqslant 4)}=1.34 \times 10^{20} \mathrm{~m}^{-3}$. That is $\sim 30 \%$ and $13.3 \%$, respectively, of the total hydrogen molecular density.

In this simple consideration, we have neglected the possibility that production may also take place via three-particle recombination or direct excitation. However, these processes would be expected to be more important in the center of the beam, where the electron density is higher, and total production is very small here. Other possibilities for production are mutual neutralization of $\mathrm{H}^{-}$atoms and dissociative recombination of $\mathrm{H}_{3}{ }^{+}$ions. The populations of these ions are expected to be negligible.

The third important feature of the lateral $n=2$ density profiles is the part of the profile outside of the plasma beam.
We used this halo in Sec. V to estimate ground-state densities outside the plasma beam.

\section{Summary of determined neutral densities}

Using electron-density and radial-dependent measurements of the $n=2$ excited population in Pilot-PSI, we have been able to obtain a rich set of information about neutrals in the plasma. In Sec. VIB 2 we found with a great deal of certainty that at high electron densities (obtained at $0.4 \mathrm{~T}$, $6.5 \mathrm{~Pa}$ ), the ground-state density in the center of the beam is $n_{\mathrm{H}}<7 \times 10^{19} \mathrm{~m}^{-3}$, giving an ionization degree of $>85 \%$. At approximately ten times higher background pressure $(50 \mathrm{~Pa})$, the ground-state density is $n_{\mathrm{H}}<3.7 \times 10^{20} \mathrm{~m}^{-3}$ and the ionization degree $>68 \%$. If we assume that the hypothesis put forward for the existence of a hot and a cold population in Sec. IV B is correct, we can estimate these values more accurately. The escape factors calculated in Sec. VI A were used to calculate neutral densities in the beam. The results were $n_{\mathrm{H}}=2.22 \times 10^{19} \mathrm{~m}^{-3}$ at $6.5 \mathrm{~Pa}$ and $1.11 \times 10^{20} \mathrm{~m}^{-3}$ at $50 \mathrm{~Pa}$, corresponding to ionization degrees of $95 \%$ and $88 \%$, respectively. These are important results as they suggest that in plasma surface interaction experiments most particles impinging on the target are ions and not neutrals.

Estimations for the neutral density outside of the beam give the dissociation degree in the vessel, which is important for modeling of the plasma and background gas system [27]. In Sec. V, the dissociation degree was estimated using a Monte Carlo model for the Lyman- $\alpha$ photons compared to $n=2$ density profiles. The approximate ground-state neutral densities were found to be $5 \times 10^{18} \mathrm{~m}^{-3}(6.5 \mathrm{~Pa})$ and $5 \times 10^{19} \mathrm{~m}^{-3}(50 \mathrm{~Pa})$, yielding a dissociation degree of $4 \%$ and $5 \%$, respectively.

The $\mathrm{H}_{2}(v \geqslant 4)$ density was estimated in Sec. VIB 2 from the peak values of the $n=2$ population density. The densities found were $3.9 \times 10^{19} \mathrm{~m}^{-3}(6.5 \mathrm{~Pa})$ and $1.34 \times 10^{20} \mathrm{~m}^{-3}$ $(50 \mathrm{~Pa})$. This corresponds to $30 \%$ and $13.3 \%$ of the total $\mathrm{H}_{2}$ density in the vessel, respectively (compared to $0.14 \%$ in Ref. [26]). In Sec. VI B 1, we saw that these percentages rise dramatically in the transition from a low electron-density, low magnetic-field regime to the high electron-density regime. At $20 \mathrm{~Pa}$ and $0.015 \mathrm{~T}$, the minimum rise in this percentage is from $<4.3 \%$ at low current to at least $19.4 \%$ at high current.

\section{DISCUSSION OF THE DIAGNOSTIC METHOD FOR DETERMINATION OF ION PARAMETERS}

The rotation velocity and temperature of ions have been measured in Sec. IV B via the $n=2$ excited neutrals. LIF is in principle an ideal method of measuring these parameters because of its high spatial resolution. It has, however, proven to be problematic in the high electron-density plasma of Pilot-PSI. The main problems are electron deexcitation that competes with the fluorescence signal, the background emission that grows by at least a factor of 10 when the magnetic-field strength is increased from 0.015 to $0.4 \mathrm{~T}$, and the hollow $n=2$ density profile at high electron densities that ensures that zero signal is measured in the center of the beam.

Absorption has proven to be a good replacement diagnostic, even though Abel inversion is required to obtain radial density 
profiles and information obtained from spectra are averages over the line of sight of the laser. Absorption ratios of down to $7 \times 10^{-4}$ have been measured and the wavelength resolution is good (spectral linewidth $<300 \mathrm{kHz}$ ). Ion velocity and temperature information could be obtained from asymmetric spectra at high electron density and high magnetic field $(0.4 \mathrm{~T})$, but could not be obtained from the symmetric spectra measured at low field $(0.015 \mathrm{~T})$ because of ambiguity in the coupling of these neutrals with ions. Figures 8 and 9 show the ion parameters obtained. Rotation velocities of $\sim 5-7 \mathrm{~km} / \mathrm{s}$ are in agreement with typical rotation velocities at a magnetic-field strength of $0.4 \mathrm{~T}$ using optical emission spectroscopy on the $\mathrm{H} \beta$ line [24]. Ion temperatures are found to be approximately equal to the electron temperatures, but because they are line-of-sight measurements, they are expected to be lower limits on the real ion temperature. Heating of the ions above the electron temperature is attributed to ion viscous heating [24].

The signal-to-noise ratio of the absorption diagnostic could be further improved by more averaging, by the use of a lock-in amplifier, and by the use of a real-time $I_{0}$ laser power measurement. This last idea would involve splitting the laser beam and guiding both beams through the vessel, with only one of the beams penetrating the plasma. A further improvement to the diagnostic could be achieved by ensuring a nonhollow $n=$ 2 density profile, such that the center of the plasma beam can be seen. The upgrade to Pilot-PSI, Magnum-PSI [4], is foreseen to have a broader plasma diameter $(\sim 10 \mathrm{~cm})$ and a lower background pressure ( $<1 \mathrm{~Pa}$ near the target). The expectation is MAR will become less important for the production of emission, first because the density of $\mathrm{H}_{2}(v \geqslant 4)$ will be lower, and second because these molecules will only be able to penetrate the edge of the beam. A larger fraction of the excited neutrals can then be expected to originate from direct electron excitation and three-particle recombination in the center.

\section{CONCLUSION}

This paper explains a methodology using $n=2$ excited atoms, for determining ion parameters and neutral densities in a linear, hydrogen, magnetized plasma with ITER divertor relevant electron parameters. Measurements were made in this regime in the beam of Pilot-PSI using LIF and absorption. Absorption was found to have a much better sensitivity than LIF, mainly owing to competition with background emission. At $4 \mathrm{~cm}$ from the source (half a meter from the target) the following parameters were measured: ion rotation velocities up to $7 \mathrm{~km} / \mathrm{s}$ and ion temperatures of $2-3 \mathrm{eV} \sim T_{e}$. Comparison of measurements with modeling yielded also the following information: a central ionization degree of $>85 \%$, a dissociation degree in the vessel of $\sim 4 \%$, and an escape factor for the Lyman- $\alpha$ line of $\sim 0.6$. Here, $30 \%$ of molecules were found to have a rovibrational excitation of more than $2 \mathrm{eV}$, a significant increase with respect to low-density, less-magnetized plasmas. The effect of a tenfold increase in pressure did not significantly affect the dissociation degree in the vessel. However, it did reduce the proportion of $v>4$ excited molecules (by $\sim 17 \%$ ) and the escape factor of the Lyman- $\alpha$ line (to $\sim 0.15$ ). In a doubling of the electron density in the transition to high electron densities, the proportion of rovibrationally excited molecules (excitation more than $2 \mathrm{eV}$ ) was found to increase by more than a factor of 4 , probably because of a large increase in the production and confinement of ground-state neutrals.

\section{ACKNOWLEDGMENTS}

This work, supported by the European Communities under the Contract of Association between EURATOM/FOM, was carried out within the framework of the European Fusion Programme with financial support from NWO.
[1] J. Roth et al., J. Nucl. Mater. 390-391, 1 (2009).

[2] H. J. van der Meiden et al., Rev. Sci. Instrum. 79, 013505 (2008).

[3] G. J. van Rooij et al., Plasma Phys. Controlled Fusion 51, 124037 (2009).

[4] H. J. N. van Eck et al., Fusion Eng. Des. 82, 1878 (2007).

[5] D. M. Goebel, G. Campbell, and R. W. Conn, J. Nucl. Mater. 121, 277 (1984).

[6] N. Ohno, N. Ezumi, S. Takamura, S. I. Krasheninnikov, and A. Yu. Pigarov, Phys. Rev. Lett. 81, 818 (1998).

[7] W. Bohmeyer, A. Markin, and C. Biedermann, Phys. Scr., T 138, 014016 (2009).

[8] S. Mazouffre et al., Phys. Rev. E 64, 016411 (2001).

[9] M. J. de Graaf, R. Severens, R. P. Dahiya, M. C. M. van de Sanden, and D. C. Schram, Phys. Rev. E 48, 2098 (1993).

[10] U. Fantz, J. Nucl. Mater. 290-293, 367 (2001).

[11] J. K. Janev, W. D. Langer, J. K. Evans, and J. D. E. Post, Elementary Processes in Hydrogen-Helium Plasmas (Springer, Berlin, 1987).

[12] P. S. Krstic, Phys. Rev. A 66, 042717 (2002).

[13] R. K. Janev, D. Reiter, and U. Samm, Tech. Rep. Jl-4105 Jül4105 (2003).

[14] W. J. Goedheer, Ph.D. thesis, Rijksuniversiteit Utrecht, 1978.

[15] J. A. M. van der Mullen and D. C. Schram, Spectrochim. Acta B 45, 233 (1990).
[16] W. A. J. Vijvers et al., Phys. Plasmas 15, 093507 (2008).

[17] K. Behringer and U. Fantz, New J. Phys. 2, 23 (2000).

[18] R. J. Rosado, Ph.D. thesis, Technische Hogeschool Eindhoven, 1981.

[19] G. J. van Rooij et al., Appl. Phys. Lett. 90, 121501 (2007).

[20] J. Westerhout, N. J. L. Cardozo, J. Rapp, and G. J. van Rooij, Appl. Phys. Lett. 95, 151501 (2009).

[21] G. M. W. Kroesen, D. C. Schram, and J. C. M. de Haas, Plasma Chem. Plasma Process. 10, 531 (1990).

[22] W. Demtröder, Laser Spectroscopy, Basic Principles (Springer, Berlin, 2008), Vol. 1.

[23] B. H. Armstrong, J. Quant. Spectrosc. Radiat. Transfer 7, 61 (1967).

[24] A. E. Shumack, V. P. Veremiyenko, D. C. Schram, H. J. de Blank, W. J. Goedheer, H. J. van der Meiden, W. A. J. Vijvers, J. Westerhout, N. J. Lopes Cardozo, and G. J. van Rooij, Phys. Rev. E 78, 046405 (2008).

[25] M. C. M. van de Sanden, Ph.D. thesis, Technische Universiteit Eindhoven, 1991.

[26] P. Vankan, D. C. Schram, and R. Engeln, Plasma Sources Sci. Technol. 14, 744 (2005).

[27] H. J. N. van Eck et al., J. Appl. Phys. 105, 063307 (2009). 